\title{
The large scale magnetic field structure of the spiral galaxy NGC 5775
}

\author{
M. Soida ${ }^{1}$, M. Krause ${ }^{2}$, R.-J. Dettmar ${ }^{3}$, and M. Urbanik ${ }^{1}$ \\ 1 Astronomical Observatory, Jagiellonian University, ul. Orla 171, 30-244 Kraków, Poland \\ e-mail: soida@oa.uj.edu.pl \\ 2 Max-Planck-Institut für Radioastronomie, Auf dem Hügel 71, 53121 Bonn, Germany \\ 3 Astronomisches Institut der Ruhr-Universität Bochum, Universitätsstr. 150, 44780 Bochum, Germany
}

Received 7 August 2008 / Accepted 25 May 2011

\begin{abstract}
Context. The origin of large-scale magnetic fields in spiral galaxies is still a theoretical riddle and better observational constraints are required to make further progress.

Aims. In order to better determine the large-scale 3D-structure of magnetic fields in spiral galaxies we present a Faraday rotation analysis of the edge-on spiral galaxy NGC 5775.

Methods. Deep radio-continuum observations in total power and linear polarization were performed at $8.46 \mathrm{GHz}$ with the VLA and the 100-m Effelsberg telescope. They were analyzed together with archival 4.86 and $1.49 \mathrm{GHz}$ VLA-data. We thus can derive rotation measures from a comparison of three frequencies and determine the intrinsic magnetic field structure.

Results. A very extended halo is detected in NGC 5775, with magnetic field lines forming an X-shaped structure. Close to the galactic disk the magnetic field is plane-parallel. The scaleheights of the radio emission esimated for NGC 5775 are comaprable with other galaxies. The rotation measure distribution varies smoothly on both sides along the major axis from positive to negative values.

Conclusions. From the derived distribution of rotation measures and the plane-parallel intrinsic magnetic field orientation along the galactic midplane we conclude that NGC 5775 has an even axisymmetric large-scale magnetic field configuration in the disk as generated by an $\alpha \Omega$-dynamo which is accompanied by a quadrupolar poloidal field. The magnetic field lines of the plane-parallel component are pointing outwards. The observed X-shaped halo magnetic field, however, cannot be explained by the action of the disk's mean-field dynamo alone. It is probably due to the influence of the galactic wind together with the dynamo action.
\end{abstract}

Key words. galaxies: magnetic fields - galaxies: spiral - galaxies: individual: NGC 5775 - radio continuum: galaxies - polarization

\section{Introduction}

The large scale structure of magnetic fields in spiral galaxies is a strong constraint for theories of the origin and evolution of cosmic magnetic fields. For external galaxies the best tracer of the magnetic field is the polarized synchrotron emission from cosmic ray electrons. For the understanding of the large scale structure of galactic magnetic fields in disk galaxies the halo field is of significant importance. In particular in view of the dynamo models which use the disk-halo interaction of the interstellar medium (ISM) to explain the growth of magnetic fields.

Due to the origin and propagation properties of cosmic ray electrons the halo magnetic field is best traced in galaxies with strong star formation in the disk. In these galaxies the star formation drives the disk-halo interaction which also allows cosmic ray electrons to escape into the halo (Dahlem et al. 2006). The propagation of cosmic ray electrons into the halo may in turn contribute to the growth of the magnetic field on large scales via e.g. fast dynamo processes (Hanasz \& Lesch 2003).

One such galaxy showing all signatures of the disk-halo interaction is the edge-on spiral NGC 5775. X-ray emission from an extended halo of hot gas (Tüllmann et al. 2006) associated with a thick disk of warm ionized $\mathrm{H}^{+}$gas (e.g. Rand 2000; Rossa \& Dettmar 2000) and a halo of synchrotron radio-continuum (Duric et al. 1998) can be traced up to $\geq 8 \mathrm{kpc}$ out of the plane.

The disk-halo interface in this object has been extensively studied (e.g. Lee et al. 2002) and in particular correlations between the radio, $\mathrm{H} \alpha$, and $\mathrm{H}$ i spurs (Collins et al. 2000) raised speculations about the role of magnetic fields in the propagating cosmic rays and the transport of ionized gas from the disk into the halo. The inclusion of magnetic reconnection (Birk et al. 1998) could also greatly relax problems with heating and ionization of the extraplanar gas (e.g. Collins \& Rand 2001b).

In a previous study (Tüllmann et al. 2000) archival VLA data at 4.86 and $1.49 \mathrm{GHz}$ were used to construct polarization maps showing the magnetic field structure in NGC 5775. The radio halo of NGC 5775 is found to be substantially polarized (polarization degree at $4.86 \mathrm{GHz}$ reaching 20-30\%) with observed polarization $B$-vectors significantly inclined to the plane (Tüllmann et al. 2000). The polarized intensity was found to make an X-shaped pattern extending along the direction of the observed $B$-vectors.

The interpretation of these observations has severe limitations: weak polarized signal at $1.49 \mathrm{GHz}$ excludes a reliable determination of Faraday rotation. In this work we present the reanalysis of the data in the context of new VLA observations at $8.46 \mathrm{GHz}$, which allow us to account for the Faraday rotation.

\section{Observations and data reduction}

The radio observations at $4.85 \mathrm{GHz}$ were already described in Tüllmann et al. (2000). New radio observations were made using the Very Large Array interferometer (VLA) of the 
Table 1. Basic properties of NGC 5775 (from LEDA database).

\begin{tabular}{lc}
\hline \hline names & NGC 5775 \\
& $\mathrm{UGC} 9579$ \\
$\mathrm{RA}_{2000}$ & $14^{\mathrm{h}} 53^{\mathrm{m}} 57^{\mathrm{s}} .6$ \\
Dec $_{2000}$ & $03^{\circ} 32^{\prime} 40^{\prime} 0$ \\
type & $\mathrm{Sbc}$ \\
incl. & $86^{\circ b}$ \\
pos angl & $145^{\circ}$ \\
distance & $26.7 \mathrm{Mpc}^{a}$ \\
& $1^{\prime} \simeq 7.8 \mathrm{kpc}$ \\
\hline
\end{tabular}

References. ${ }^{a}$ Dahlem et al. (1995); ${ }^{b}$ Irwin (1994).

Table 2. Rms noise level $(\sigma)$ obtained in final total power and polarized intensity maps.

\begin{tabular}{lcccccc}
\hline \hline Freq. & $\begin{array}{c}\sigma \text { (total } \\
\text { power) } \\
{\left[\frac{\mu \mathrm{Jy}}{\text { b.a. }}\right]}\end{array}$ & $\begin{array}{c}\sigma(\text { Stokes } \\
Q \text { and } U) \\
{\left[\frac{\mu \mathrm{Jy}}{\text { b.a. }}\right]}\end{array}$ & $\begin{array}{c}\sigma(\text { pol } \\
\text { int.) } \\
{\left[\frac{\mu \mathrm{Jy}}{\text { b.a. }}\right]}\end{array}$ & $\begin{array}{c}\text { Beam } \\
\text { size } \\
{\left[{ }^{\prime \prime}\right]}\end{array}$ & \\
{$[\mathrm{GHz}]$} & 7 & 8 & 9 & 16 & D-array \\
8.46 & 13 & 70 & 72 & 75 & 84 & Effelsberg \\
8.35 & 400 & 70 & 7 & 7 & 16 & D-array \\
4.86 & 10 & 6 & 23 & 23 & 16 & C\&D-array \\
1.49 & 60 & 23 & 25 & & & \\
\hline
\end{tabular}

National Radio Astronomical Observatory $\left(\mathrm{NRAO}^{1}\right)$. The data at $8.46 \mathrm{GHz}$ were collected using the most compact (D-array) configuration for the best sensitivity to extended emission. Deep continuum observations (about $13 \mathrm{~h}$ on source) with full Stokes parameters were recorded. Data at other frequencies were taken from the NRAO VLA archive from various observation projects. A total of $10 \mathrm{~h}$ on-source time at $4.86 \mathrm{GHz}$ (in D-array), and 14 and $2.5 \mathrm{~h}$ at $1.49 \mathrm{GHz}$ (in $\mathrm{D}$, and $\mathrm{C}$-array respectively) were found useful for a polarimetry study.

All interferometric data were reduced using the AIPS data processing package. The nearby point source $1442+101$ was used for phase and instrumental polarization calibration. The source 3C 286 was used for calibrating flux density scale and polarization position angle.

Complementary single-dish mapping of the galaxy was made with the 100-m Effelsberg ${ }^{2}$ radio telescope. The Effelsberg $\lambda 3.6 \mathrm{~cm}(8.35 \mathrm{GHz})$ observations were made with a single-beam receiver in the secondary focus of the 100-m telescope. The receiver has two canals (RHC, LHC) with total-power amplifiers and an IF polarimeter. The bandwidth was $1.1 \mathrm{GHz}$, the system noise temperature about $25 \mathrm{~K}$, and the resolution $84^{\prime \prime}$ HPBW.

We obtained 33 coverages in total of NGC 5775 between February and July 2003. Each coverage has a map size of $18^{\prime} \times 18^{\prime}$ and was scanned alternatively along RA and Dec directions. The scanning velocity was $30^{\prime \prime} / \mathrm{s}$ and the grid size is $30^{\prime \prime}$.

For the pointing and focusing we observed regularly the source 3C 286. The flux calibration was also done with 3C 286 according to the flux values of Baars et al. (1977). All coverages were combined (Emerson \& Gräve 1979) separately for the Stokes parameters $I, Q$, and $U$. The rms noise levels of the final maps are given in Table 2.

\footnotetext{
1 NRAO is a facility of National Science Foundation operated under cooperative agreement by Associated Universities Inc.

2 Effelsberg 100-m telescope is operated by Max-Planck-Institut für Radioastronomie in Bonn (MPIfR) on behalf of the Max-PlanckGeselschaft.
}

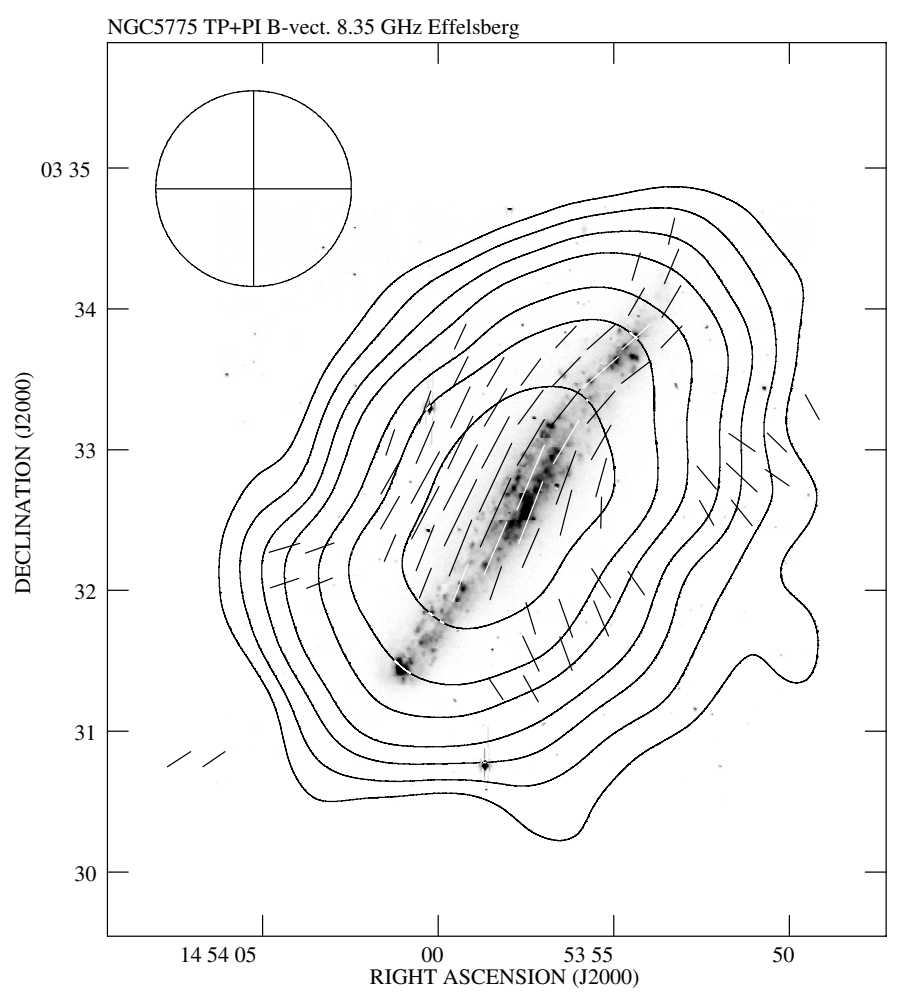

Fig. 1. Total intensity map at $8.35 \mathrm{GHz}$ (contours)with polarization observed with the 100-m Effelsberg telescope, superimposed on the $\mathrm{H} \alpha$ image (from Tüllmann et al. 2000). The contour levels are (3, 5, 8, $12,20,30,50) \times 400 \mu \mathrm{Jy} /$ b.a. (rms). The vectors show the apparent $B$-orientation, their lengths are proportional to the polarized intensity ( $1^{\prime}$ corresponds to $1.2 \mathrm{mJy} / \mathrm{b}$.a.). The angular resolution is $84^{\prime \prime} \mathrm{HPBW}$.

The single-dish data at $8.35 \mathrm{GHz}$ were merged with the interferometric ones providing zero-spacing information, that is missing in the interferometric data alone. The merging was performed in the Fourier plane (AIPS task IMERG) with the Effelsberg map scaled in intensity to the VLA observing frequency, assuming a constant spectral index of $\alpha=0.85\left(S_{v} \propto\right.$ $\left.v^{-\alpha}\right)$. About $3 \%$ of the total intensity flux was recovered with this procedure. Complimentary single-dish observations were made also at $4.85 \mathrm{GHz}$ using the $100-\mathrm{m}$ Effelsberg telescope. Both single-dish and interferometric data had the same integrated total flux and so no merging was performed at this frequency.

The maps in all Stokes parameters $(I, Q$, and $U)$ were convolved to a common beam with HPBW of $16^{\prime \prime}$ and combined to obtain final maps of total and polarized intensities and polarization magnetic angles $\left(E\right.$-vectors rotated by $\left.90^{\circ}\right)$ called hereafter as "apparent magnetic field orientations" (in short: apparent $B$ orientation).

\section{Results}

\subsection{Single-dish data}

The total intensity map obtained with the 100-m Effelsberg telescope alone at $8.35 \mathrm{GHz}$ is presented in Fig. 1 along with polarization $B$-vectors that give the apparent magnetic field orientation (i.e. without correction for Faraday rotation, which is small at such a high frequency) projected to the sky plane. The intensity distribution shows a small asymmetry with respect to the disk plane of the galaxy, being more extended to the southwestern side of the disk. Two extensions can be noticed on the southern and eastern side of the galaxy (on both sides of the disk 


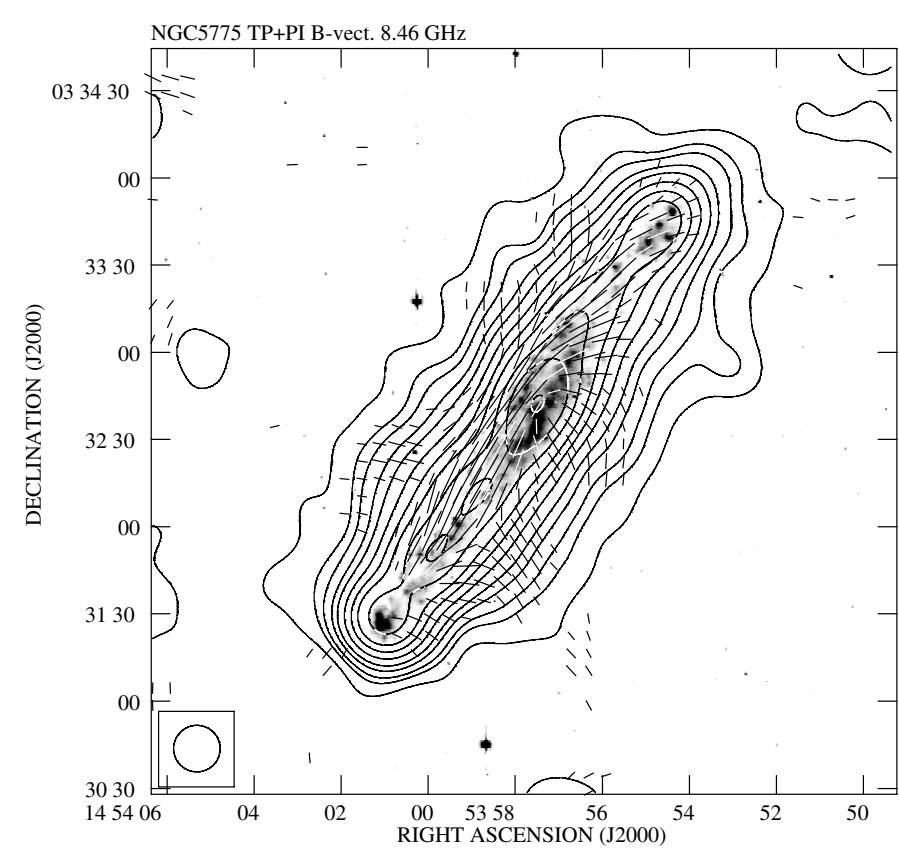

Fig. 2. Total intensity contour map with apparent polarization $B$-vectors at $8.46 \mathrm{GHz}$ (combined VLA + Effelsberg) superimposed on the $\mathrm{H} \alpha$ image (from Tüllmann et al. 2000). Contours are plotted at levels (3, 5, $8,12,20,30,50,80,120,200,300$ and 500) $\times 13 \mu \mathrm{Jy} / \mathrm{b}$.a. (rms level this frequency). Vectors are proportional to the polarized intensity and indicate apparent $B$-orientation (10" corresponds to $100 \mu \mathrm{Jy} / \mathrm{b}$.a.). The resolution is $16^{\prime \prime} \mathrm{HPBW}$.

plane) both accompanied by polarization magnetic vectors at an angle of about $45^{\circ}$ to the plane. The apparent magnetic field orientations close to the disk are plane parallel along the whole radial extent of the disk. The 84 " beam "smoothes" the diskparallel orientation of the magnetic vectors to the north-eastern side of the disk plane. Further away from the disk, the vectors are nearly perpendicular to the disk in the western side of the disk plane. The gap in the distribution of the polarization vectors on the western part, between the two predominant orientations of the vectors, is clearly caused by beam depolarization: the magnetic field changes its direction by about $90^{\circ}$ within the large single-dish beam.

\subsection{High-resolution total intensity data}

The total intensity distributions of NGC 5775 at three radio wavelengths $(8.46 \mathrm{GHz}, 4.86 \mathrm{GHz}$ and $1.49 \mathrm{GHz})$ and the corresponding apparent magnetic vectors (with lengths proportional to polarized intensity) are presented in Figs. 2 and 3 overlaid on the $\mathrm{H} \alpha$ distribution (from Tüllmann et al. 2000). The distribution is quite similar at all three frequencies, showing smooth maxima of emission clearly corresponding to the star-forming concentrations traced by the $\mathrm{H} \alpha$ emission. No evidence is seen for any violent nuclear activity.

Some weak extensions in all four quadrants are seen, best visible in the 8.46 and $4.86 \mathrm{GHz}$ maps (Figs. 2 and 3 left). The extensions become obvious after some median filtering is applied (see Fig. 5 in Tüllmann et al. 2000).

A very extended radio halo, which can be traced up to more than $1^{\prime}$ from the galactic disk is seen in all maps. Assuming the distance to the galaxy of $26.7 \mathrm{Mpc}$ (Dahlem et al. 1995) this corresponds to about $8 \mathrm{kpc}$. We want to stress here, that this is the extension of the halo as visible given by the noise-level of our observations and not the scale height of the radio emission, which is, of course, smaller (see Sect. 4.1).

Flux integrations in elliptical rings corresponding to the shape of the galaxy give total flux densities of NGC 5775 of $58 \pm 3 \mathrm{mJy}$ at $8.46 \mathrm{GHz}, 96 \pm 5 \mathrm{mJy}$ at $4.86 \mathrm{GHz}$ and $257 \pm 13 \mathrm{mJy}$ at $1.49 \mathrm{GHz}$. These values fit well a single power-law with a spectral index of $\alpha=0.85 \pm 0.02$ (where $S_{v} \propto v^{-\alpha}$ ), which is in agreement with earlier works (Duric et al. 1998). As the spectrum looks a bit flattened at the highest frequency we estimated a mean nonthermal spectral index of $\alpha_{\mathrm{nt}}=0.92$ and, correspondingly, a mean thermal fraction of $\sim 9 \%$ at $1.49 \mathrm{GHz}$, rising to $\sim 15 \%$ at $8.46 \mathrm{GHz}$.

\subsection{High-resolution polarization data}

Figure 4 shows the polarized intensity (contours) with vectors of apparent magnetic field orientation at $8.46 \mathrm{GHz}$ and proportional to the degree of linear polarization (and overlaid again on the $\mathrm{H} \alpha$ map). The contours of linearly polarized intensity at $4.86 \mathrm{GHz}$ are shown in Fig. 5, together with polarization B-vectors corrected for Faraday rotation (see also Sect. 4.6). The degree of polarization does not exceed $10 \%$ close to the disk plane and reaches $40 \%$ in the outskirts of the galaxy.

The extensions in the four quadrants as described in Sect. 3.2 are clearly visible in the polarized intensity maps, especially at $4.86 \mathrm{GHz}$ (Fig. 5) as this map has the highest signal-to-noise ratio. The polarized intensity seems to form an X-shaped structure.

The apparent magnetic field orientation at $8.46 \mathrm{GHz}$ (Fig. 4) close to the galactic disk is plane parallel on the north-eastern side of the disk. Vectors are turning rapidly (within a single beam-size area) when crossing the disk and become almost perpendicular on the other side of the disk plane. Such a configuration gives rise to a narrow beam-depolarized canal along the south-western edge of the galactic disk (from RA: $14^{\mathrm{h}} 53^{\mathrm{m}} 59^{\mathrm{s}}$; Dec: $+03^{\circ} 32^{\prime} 00^{\prime \prime}$ to RA: $14^{\mathrm{h}} 53^{\mathrm{m}} 57^{\mathrm{s}}$; Dec: $\left.+03^{\circ} 32^{\prime} 50^{\prime \prime}\right)$. The depolarized canal is even better seen at $4.86 \mathrm{GHz}$ (Fig. 5) and not seen at $1.49 \mathrm{GHz}$ due to the overall weak polarized signal at this frequency.

The extensions in polarized intensity and total power are accompanied with vectors of apparent $B$-configuration that are also oriented at an angle of $\sim 45^{\circ}$ with respect to the galactic plane, forming the $X$-shaped structure. The coincidence of polarized intensity with total power extensions is best visible in the higher frequency maps (Fig. 2 and Fig. 3 left panel) but is still indicated at the noise limit in the $1.49 \mathrm{GHz}$ map (Fig. 3 right).

The apparent magnetic field configuration is very similar at 8.46 GHz and $4.86 \mathrm{GHz}$ (Fig. 2 and Fig. 3 left). This already allows us to conclude that Faraday effects are not very strong in NGC 5775 at these short wavelengths. At $1.49 \mathrm{GHz}$ the polarized emission is detected in isolated patches only and if compared to the higher frequency maps the observed polarization orientation at this frequency is clearly Faraday-rotated close to the disk plane. However, in the outskirts of the galaxy, the apparent magnetic vectors even at $1.49 \mathrm{GHz}$ (where detected) agree well with those at higher frequencies, suggesting only small Faraday effects there.

\section{Discussion}

\subsection{Total power emission and vertical scale heights}

The intensity distribution of the total power along the major axis of the galaxy at $4.86 \mathrm{GHz}$ (Fig. 3, left) is shown in Fig. 6. 

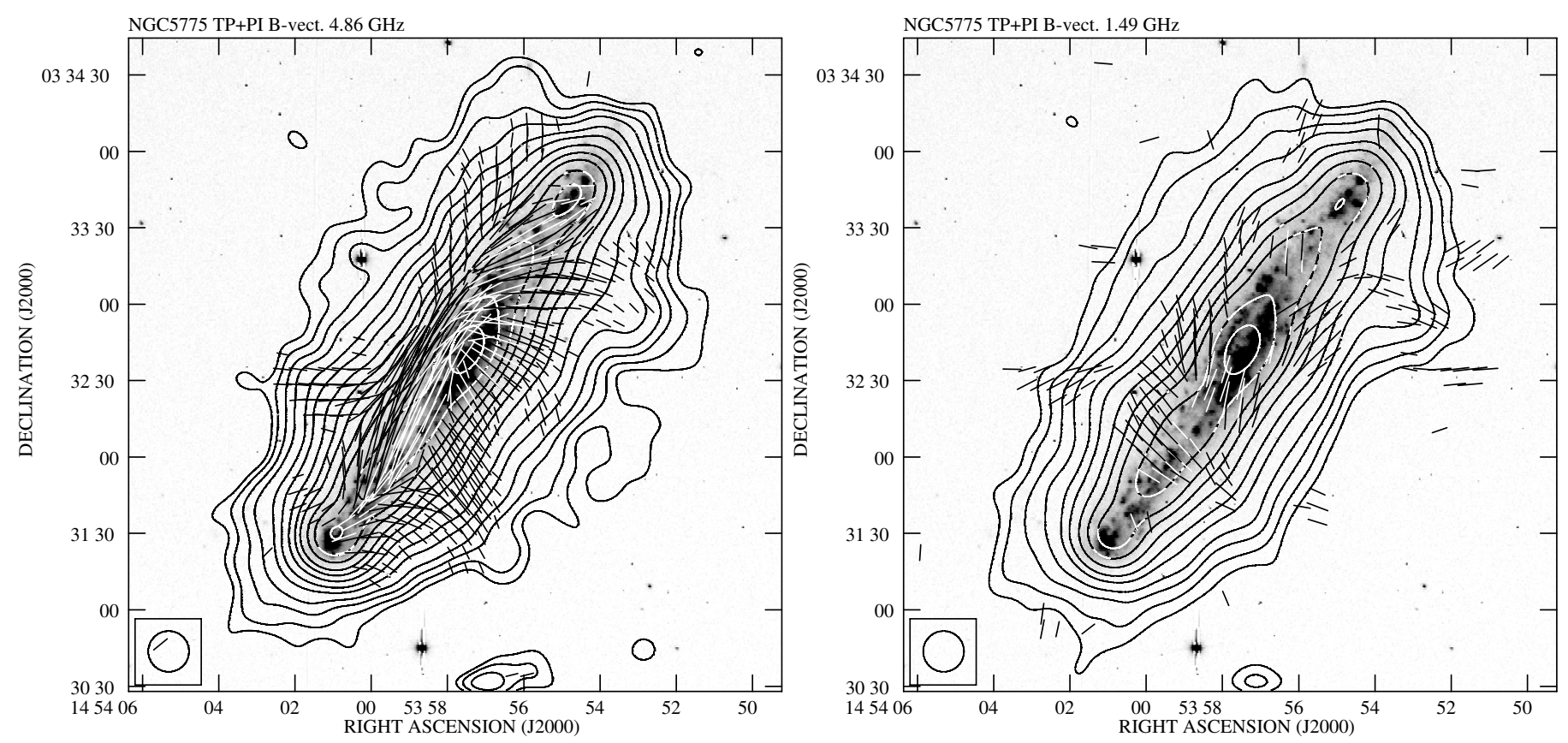

Fig. 3. Total intensity contour map with apparent polarization $B$-vectors at $4.86 \mathrm{GHz}$ (left) and $1.49 \mathrm{GHz}$ (right). Both maps are superimposed on the $\mathrm{H} \alpha$ image (from Tüllmann et al. 2000). Contours are plotted at levels (3, 5, 8, 12, 20, 30, 50, 80, 120, 200, 300 and 500$) \times 10$ and $60 \mu \mathrm{Jy} / \mathrm{b} . \mathrm{a}$., respectively (rms levels at corresponding frequencies). Vectors are proportional to the polarized intensity (10" corresponds to $50 \mu \mathrm{Jy} / \mathrm{b} . \mathrm{a}$.) and indicate apparent $B$-orientation. The resolution is $16^{\prime \prime}$ HPBW in both maps.

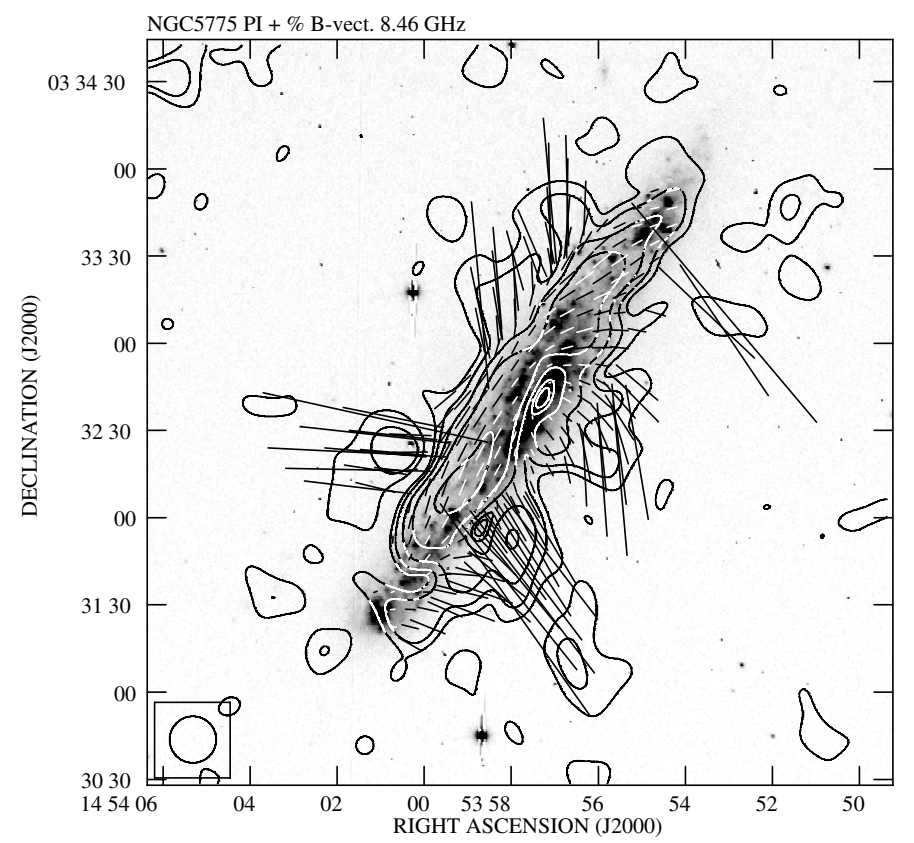

Fig. 4. Polarized intensity contour map at $8.46 \mathrm{GHz}$ with apparent polarization $B$-vectors, superimposed on the $\mathrm{H} \alpha$ image (from Tüllmann et al. 2000). Contours are plotted at levels $(3,5,8,12$, and 20$) \times 9 \mu \mathrm{Jy} / \mathrm{b}$.a. (rms). Vectors are proportional to the degree of polarization $\left(10^{\prime \prime}\right.$ corresponds to $10 \%$ ) and show the apparent $B$-orientation. Only vectors above $3 \sigma$ rms level (in both total and polarized emission) are shown. The resolution is $16^{\prime \prime} \mathrm{HPBW}$.

The central maximum is slightly shifted to the northern part of the galaxy with a steep decrease further north, but a slower decrease with a plateau along the southern part of the major axis. The outermost local maxima on both sides of the major axis are roughly at the same distance from the nucleus $\left(r=80^{\prime \prime}\right.$ or

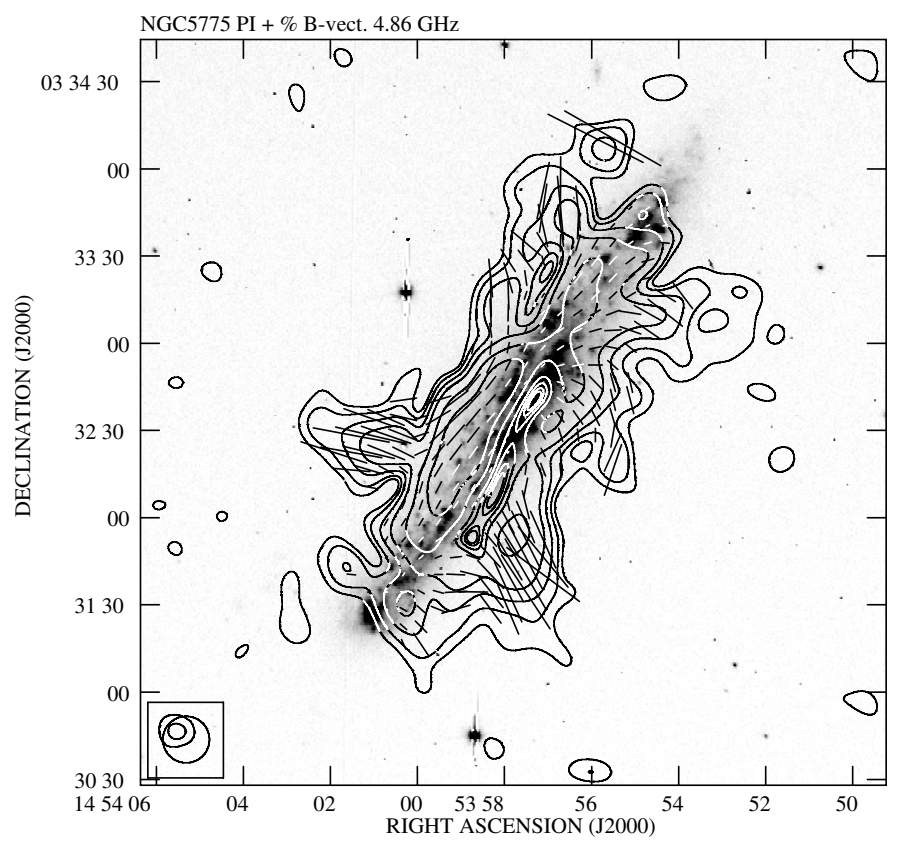

Fig. 5. Polarized intensity contour map at $4.86 \mathrm{GHz}$ with intrinsic (Faraday-corrected) magnetic field orientation, superimposed on the $\mathrm{H} \alpha$ image (from Tüllmann et al. 2000). Contours are plotted at levels (3, $5,8,12,20$ and 30$) \times 7 \mu \mathrm{Jy} / \mathrm{b}$.a. $(\mathrm{rms})$. Vectors are proportional to the degree of polarization (10" corresponds to $10 \%)$. Vector plotting was suppressed below $3 \sigma \mathrm{rms}$ level (in both total and polarized emission). The resolution is $16^{\prime \prime} \mathrm{HPBW}$.

$10.7 \mathrm{kpc}$ ) and probably indicate the positions of the outer spiral arms. The observed vertical extension of the total power emission is due to the real disk thickness and the inclined distribution of the radial disk emission, smoothed by the telescope beam. In order to estimate the latter effect, we projected the radial total 


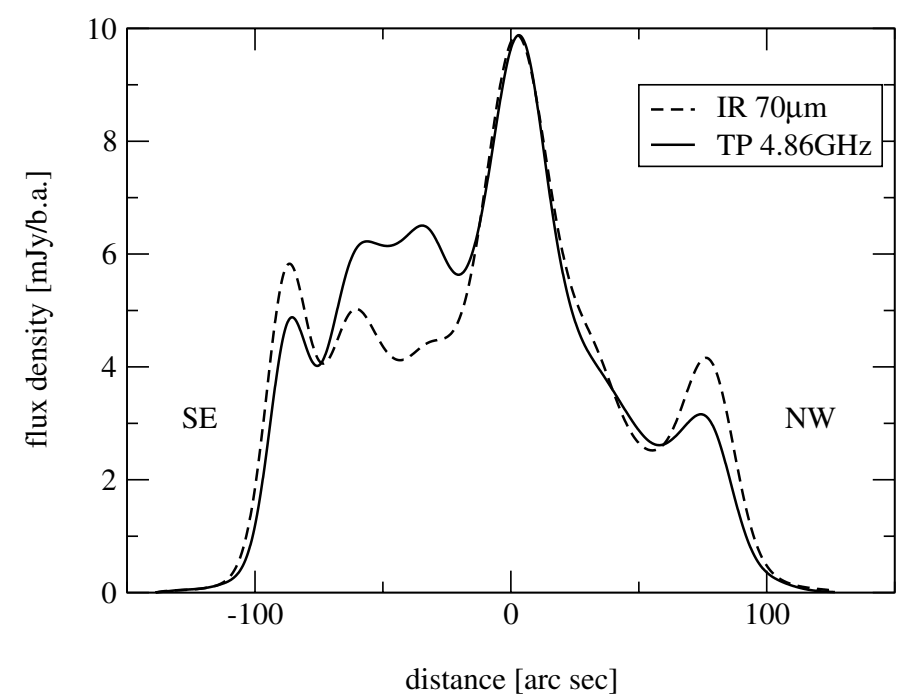

Fig. 6. Total intensity distribution at $4.86 \mathrm{GHz}\left(16^{\prime \prime} \mathrm{HPBW}\right)$ along the major axis of NGC 5775. The $x$-axis gives the distance from the nucleus of the galaxy.

Table 3. Vertical scale heights for the thin and thick disk.

\begin{tabular}{lcccccc}
\hline \hline & \multicolumn{3}{c}{$8.46 \mathrm{GHz}$} & \multicolumn{2}{c}{$4.86 \mathrm{GHz}$} & \multicolumn{2}{c}{$1.49 \mathrm{GHz}$} \\
Strip & \multicolumn{2}{c}{$h_{\text {thin }}$} & $h_{\text {thick }}$ & $h_{\text {thin }}$ & $h_{\text {thick }}$ & \multicolumn{2}{c}{$h_{\text {thin }}$} & $h_{\text {thick }}$ \\
& {$[\mathrm{pc}]$} & {$[\mathrm{kpc}]$} & {$[\mathrm{pc}]$} & {$[\mathrm{kpc}]$} & {$[\mathrm{pc}]$} & {$[\mathrm{kpc}]$} \\
\hline $\mathrm{n} 1$ & 250 & 3.4 & 240 & 1.9 & 238 & 2.0 \\
$\mathrm{n} 2$ & 301 & 2.4 & 202 & 1.7 & 228 & 1.9 \\
$\mathrm{~s} 1$ & 243 & 2.7 & 237 & 2.2 & 268 & 1.7 \\
$\mathrm{~s} 2$ & 320 & 1.8 & 295 & 2.0 & 286 & 1.5 \\
\hline mean & 279 & 2.6 & 244 & 2.0 & 255 & 1.8 \\
& \pm 33 & \pm 0.6 & \pm 33 & \pm 0.2 & \pm 24 & \pm 0.2 \\
\hline
\end{tabular}

power distribution (Fig. 6) to the inclination of the galaxy of $86^{\circ}$ and convolved this distribution with a Gaussian function with $16^{\prime \prime}$ HPBW to simulate the effect of the telescope beam. The HPBW of the resulting distribution is $21.3^{\prime \prime}$ which is called the effective beam size. Only the parts of the emission profiles perpendicular to the disk that exceed the effective beam size can be attributed to extra-planar emission.

We determined the vertical scaleheights from emission profiles that were obtained by strip integration perpendicular to the major axis along 3 strips with a width of $60^{\prime \prime}(7.8 \mathrm{kpc})$ each, centered on the nucleus. These profiles were fitted with a model distribution (for details see Dumke et al. 1995) consisting of an intrinsic two-component exponential profile convolved with the effective beam size. The fits were made separately for the emission "above" (north $=\mathrm{n}$ ) and "below" ( midplane and at all three wavelengths.

The values for both the thin and thick disks in $\mathrm{n} 3$ are significantly larger than in the other strips, and also those in s3 deviate strongly from the values in strips 1 and 2 . According to Tüllmann et al. (2006) there is most likely a background galaxy cluster along the line of sight through strip n3, and possibly also along strip s3. This may influence the measured values at these positions, so that we cannot regard them as being scaleheights of NGC 5775.

The results for strips 1 and 2 are summarized in Table 3 where the strip number 1 refers to a radius $-90^{\prime \prime}$ to $-30^{\prime \prime}$ and strip number 2 to $-30^{\prime \prime}$ to $30^{\prime \prime}$ along the major axis (from southeast to north-west).
The averages of the scaleheights in Table 3 are given as mean with errors reflecting the variations of the scaleheights between the single strips. The $4.86 \mathrm{GHz}$ values for the thin $(240 \pm 30 \mathrm{pc})$ as well for the thick disk $(2.0 \pm 0.2 \mathrm{kpc})$ agrees within their errors with the scaleheights observed in four other edge-on galaxies at this wavelength (Dumke \& Krause 1998; Heesen et al. 2009). This sample of four galaxies includes NGC 253 with the brightest known halo and a very high star formation rate (SFR) as well as one with the weakest halo, NGC 4565, with a small SFR. The star formation in NGC 5775 is comparably strong as that of NGC 253. The observed scaleheight in NGC 5775 supports our previous result that a strong star formation does not increase the global scaleheight of the radio emission (Krause 2009).

As shown in Table 3 the thin-disk vertical scaleheights as well as the scaleheights for the thick disk agree with each other within their errors. Synchrotron losses decrease the vertical scale heights with increasing frequencies. On the other hand, the thermal fraction increases with frequency. The observed similarity of scaleheights at all three frequencies may be due to a superposition of both effects which also implies that the thermal emission is not confined to the thin disk.

The analysis of the radial dependence of the scaleheight at one frequency of NGC 253 showed indeed (together with spectral aging effects) a vertical cosmic ray (CR) transport from the disk into the halo (Heesen et al. 2009). There, the CR lifetime is dominated by synchrotron losses which depend on the magnetic field strength and is highest in the central region. This leads to smallest scaleheights in the central part and increasing values for larger radii visible as a dumbbell shape of the halo. This effect is also visible in NGC 5775: strip1 has larger values for the thick disk than strip2 (Table 3). However, the smaller angular extent of NGC 5775 (which could not be compensated by higher angular resolution of the observations) compared to NGC 253 and the contamination by the background galaxy cluster in strip3 made a further analysis impossible.

\subsection{Spectral index distribution}

The spectral index distribution was calculated using all three total intensity maps as a fit of a power-law spectrum to each point and is shown in Fig. 7 (together with polarization $B$-vectors at $4.86 \mathrm{GHz}$ ). The spectrum is flattest along the disk and especially on $\mathrm{H} \alpha$ concentrations ( $\sim 0.5)$ and steepens smoothly (up to $\sim 1.3$ ) towards the outer boundary of the halo. The steepening seems to be slightly stronger towards the north-eastern boundary ("above" the disk) than towards the south-western boundary ("below" the disk) where even a slight flattening is indicated just west of the nucleus which coincide with extended X-ray emission as shown in Tüllmann et al. (2006). Some steepening of the spectrum can be noticed along with all four polarized intensity extensions, which may reflect slightly faster synchrotron losses in the locally enhanced magnetic field there.

Our spectral index map made of three frequency observations cannot confirm the vertical structures (chimneys) of flattened spectral indices as presented by Duric et al. (1998) who determined the spectral index map from the VLA observations at $4.86 \mathrm{GHz}$ and $1.49 \mathrm{GHz}$ alone. This can hardly be due to the slightly higher angular resolution of their map $\left(15^{\prime \prime} \times 13^{\prime \prime}\right)$ compared to our 16" HPBW map but, as we use a larger dataset (all available in the VLA archive), hence it may be artifacts in their maps.

In order to quantify the spectral index variations we determined the spectral index between all 3 frequencies in the same 3 strips described in Sect. 4.1. The values are presented in Fig. 8. 


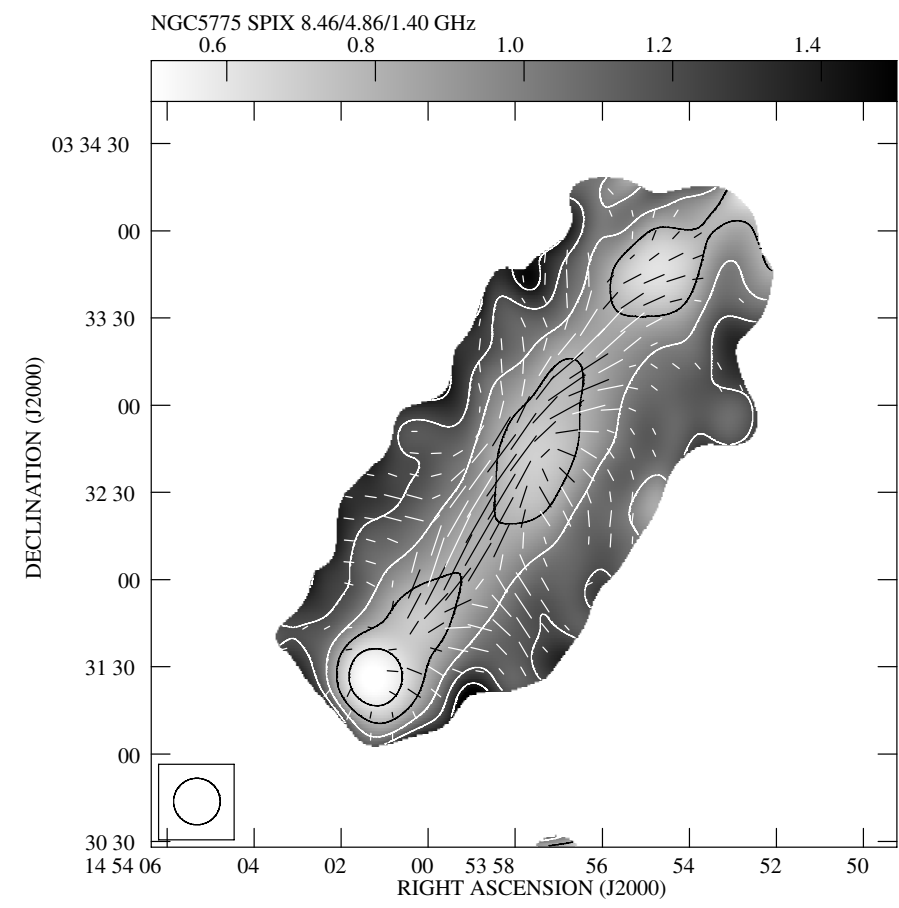

Fig. 7. Spectral index map calculated using all the data at $8.46 \mathrm{GHz}$, $4.86 \mathrm{GHz}$ and $1.49 \mathrm{GHz}$. Contours are of the same quantity, plotted at $\alpha=0.6,0.8$ (black) and 1.0, 1.2, 1.4 (white) levels $\left(S_{v}=S v^{-\alpha}\right)$. The resolution is $16^{\prime \prime} \mathrm{HPBW}$. Magnetic vectors proportional to the polarized intensity at $4.86 \mathrm{GHz}$ are overplotted $\left(10^{\prime \prime}\right.$ corresponds to $100 \mu \mathrm{Jy} / \mathrm{b}$.a.).

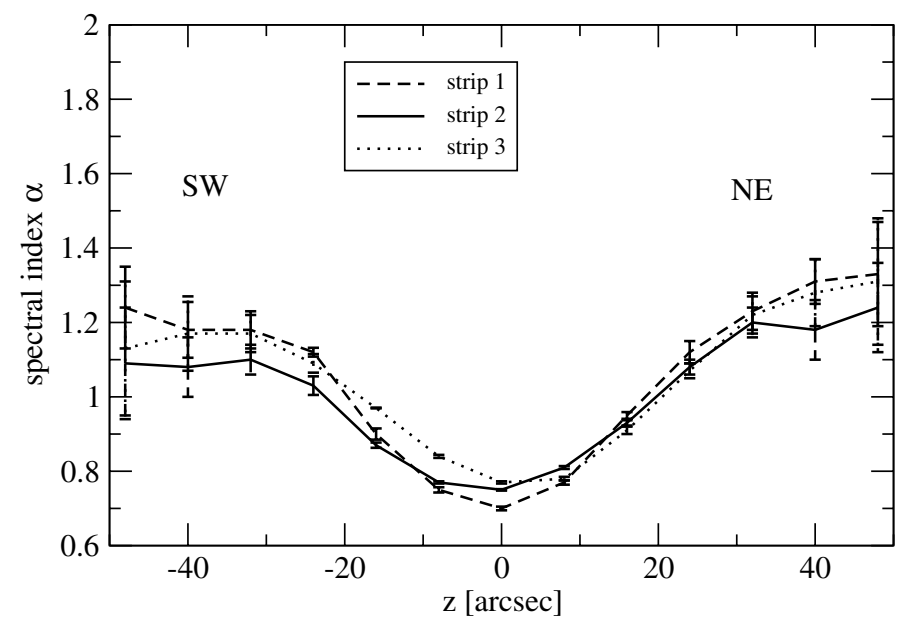

Fig. 8. Spectral index distribution within three strips perpendicular to the major axis: strip 1 (dashed) refers to a radius $-90^{\prime \prime}$ to $-30^{\prime \prime}$, strip 2 (full) from $-30^{\prime \prime}$ to $30^{\prime \prime}$, and strip 3 (dotted) from $30^{\prime \prime}$ to $90^{\prime \prime}$ along the major axis (from south-east to north-west).

The spectra increase in all strips from the midplane with values of about $\alpha=0.75$ up to $\alpha=1.1$ at 32 arcsec distance on both sides away from the midplane. Such a steepening is expected by a decrease of the thermal fraction from the midplane to higher $z$-values. Additionally, synchrotron losses of the nonthermal radiation are expected to increase with increasing distance from the midplane. As the highest reliable values for the spectral indices are about $\alpha=1.1$ and the value for the mean nonthermal spectral index $\alpha_{\mathrm{nt}}=0.92$ (as estimated in Sect. 3.2). Assuming a thermal content in the disk of about $11 \%$ at $1.49 \mathrm{GHz}$ (equivalent to about $35 \%$ at $8.46 \mathrm{GHz}$ ), we can explain the spectrum

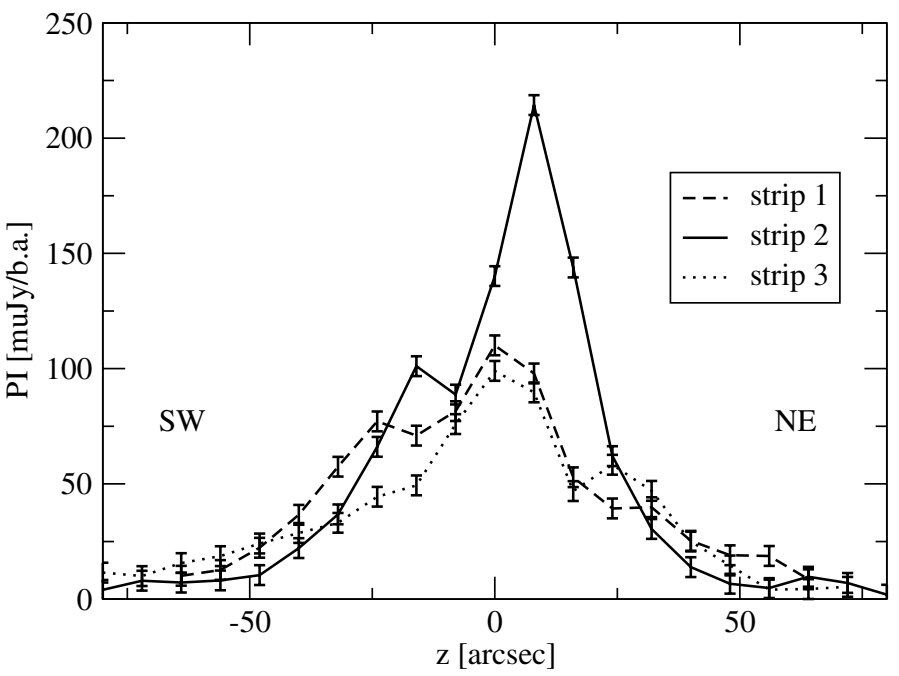

Fig. 9. Polarized intensity at $4.86 \mathrm{GHz}$ averaged along three strips of $60^{\prime \prime}$ width each, perpendicular to the major axis: strip 1 (dashed) refers to a radius $-90^{\prime \prime}$ to $-30^{\prime \prime}$, strip 2 (full) from $-30^{\prime \prime}$ to $30^{\prime \prime}$, and strip 3 (dotted) from $30^{\prime \prime}$ to $90^{\prime \prime}$ along the major axis (from south-east to northwest).

flattening from the mean $\alpha_{\mathrm{nt}}=0.92$ down to $\alpha=0.75$. The further steepening - up to $\alpha=1.1$ - we can attribute to synchrotron losses at the galactic peripheries.

The spectral index distribution in strip 3 (dotted) is slightly shifted to positive $z$-values with respect to the distributions in the two other strips. This shift seems to be significant as the errors are very small in the central parts. It may be due to the background galaxy cluster in strip3 as discussed in Sect. 4.1.

Even in the strip integration we see an indication of a spectral index flattening in the central strip (full line) for $z \leq-30$ " ("below" the disk) where the X-ray emission is strongest (Tüllmann et al. 2006).

\subsection{Vertical distribution of the polarized intensity}

As described in Sect. 3.2 the region with the magnetic field plane parallel to the galactic disk is slightly shifted to the north-east ("upper") part of the disk (best visible in Fig. 2). We wanted to test whether this asymmetry in the distribution of magnetic field orientation is also accompanied by an asymmetry in the distribution of the polarized intensity. Therefore we integrated also the polarized intensity at $4.86 \mathrm{GHz}$ in the same three strips parallel to the minor axis as described in Sect. 4.1. The result is shown in Fig. 9.

The maximum of the polarized intensity of the central strip (full line) is clearly shifted to positive $z$-values (north-east), the maxima in the two other strips are slightly shifted towards the same direction. All maxima agree in position with the regions of the plane-parallel magnetic field orientation. Further, all distributions of polarized intensity are asymmetric in shape: they show a rather strong decrease with increasing $|z|$, but have a shoulder towards negative $z$-values where the magnetic field orientation is mainly vertical to the galactic plane.

From X-ray observations (e.g. Tüllmann et al. 2006) we can conclude that the north-eastern side of NGC 5775 is the near side, hence we see the disk slightly from "below". This can explain why we see the disk-parallel magnetic field which is supposed to be strongest, somewhat shifted to the north-east with respect to the galactic midplane (see discussion in Sect. 4.6). 


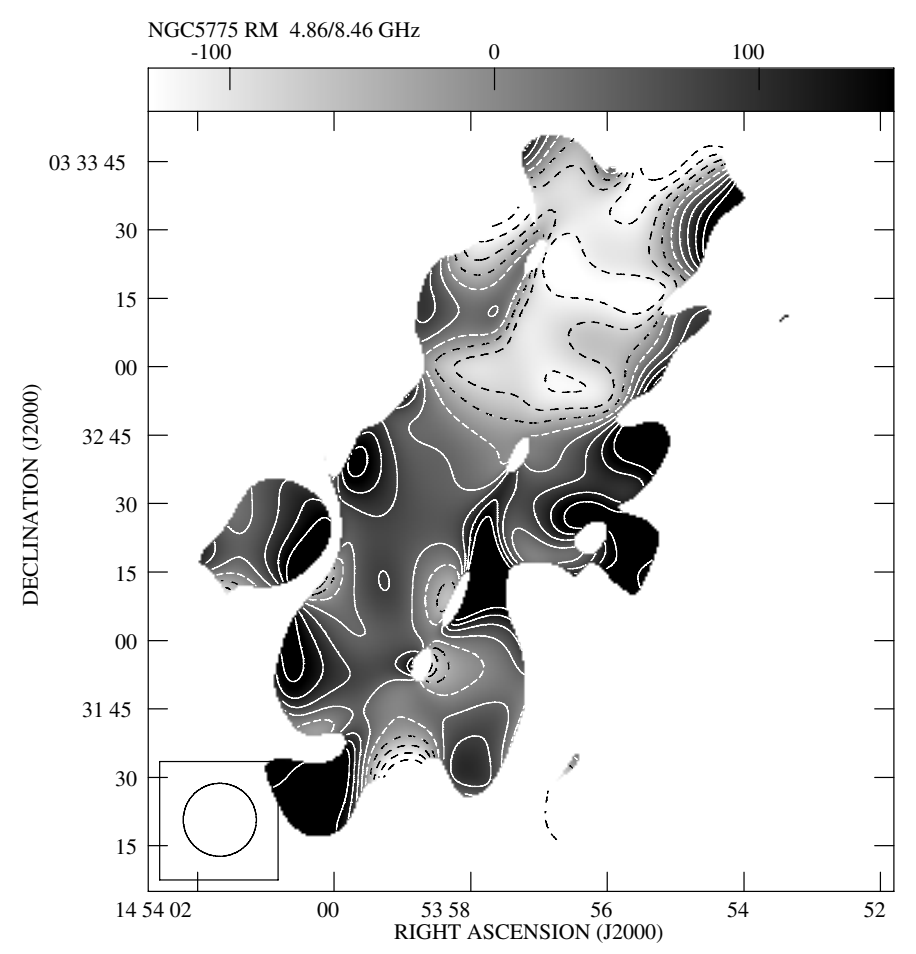

Fig. 10. The Faraday rotation measure distribution determined between $8.46 \mathrm{GHz}$ and $4.86 \mathrm{GHz}$ maps. Contours are plotted in RM intervals of $40 \mathrm{rad} / \mathrm{m}^{2}$ - the dashed white contour corresponds to the RM value of $0 \mathrm{rad} / \mathrm{m}^{2}$. The resolution is $16^{\prime \prime} \mathrm{HPBW}$.

\subsection{Faraday rotation and depolarization}

The Faraday rotation measure distribution, calculated between $8.46 \mathrm{GHz}$ and $4.86 \mathrm{GHz}$, is presented in Fig. 10 in gray-scales with contours - white and solid for positive values, black and dashed for negative ones. The distribution was determined from data truncated at the $3 \sigma$ (rms) levels in the polarized intensity maps resulting in a maximal uncertainty at the limits of detected polarized signal of about $100 \mathrm{rad} / \mathrm{m}^{2}$.

The overall rotation measure (RM) has values between $\pm 140 \mathrm{rad} / \mathrm{m}^{2}$ and exceeds this range only in small isolated areas where uncertainties are substantial. Its distribution is well balanced among positive and negative values. This suggests a negligible foreground Faraday rotation at the position of NGC 5775. This is in agreement with measurements of, e.g., Taylor et al. (2009).

There is a clear trend along the major axis: RM is negative in the north-western and positive in the south-eastern half of the galaxy. Two jumps in RM distribution (close to position of RA: $14^{\mathrm{h}} 53^{\mathrm{m}} 58^{\mathrm{s}}$; Dec: $+03^{\circ} 32^{\prime} 00^{\prime \prime}$ ) are caused by large uncertainty of RM determination in locations with weak polarized intensity (compare with polarized intensity maps, particularly at 4.86 GHz in Fig. 5).

The Faraday depolarization was determined as the ratio of the polarization degree at $4.86 \mathrm{GHz}$ to that at $8.46 \mathrm{GHz}$ and is presented in Fig. 11. The data were clipped at the $3 \sigma$ level in total and polarized intensity prior to the determination of this this quantity. We decided to use the data at the two higher frequencies since the $1.49 \mathrm{GHz}$ polarized emission exceeds the cutting level only in very limited area. The distribution is smooth, with mean values of $60-80 \%$. Some axial symmetry is visible with higher values (less depolarization) found close to the rotation axis in agreement with the Faraday rotation distribution,

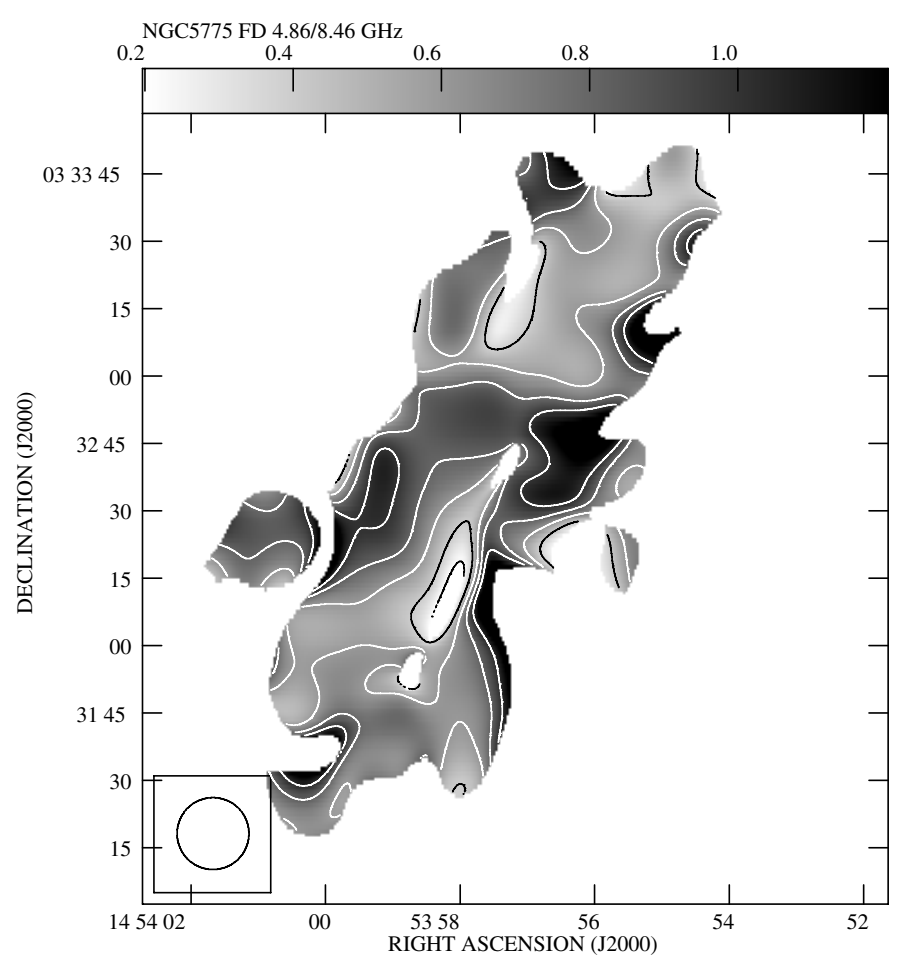

Fig. 11. Depolarization map calculated as the ratio of polarization degree at $4.86 \mathrm{GHz}$ to that at $8.46 \mathrm{GHz}$. Contours are of the same quantity, plotted at 1.0, 0.8, 0.6 (white) and 0.4 and 0.2 (black) levels. The resolution is $16^{\prime \prime}$ HPBW. The data were clipped at the $3 \sigma$ level in total and polarized intensity prior to the determination of this this quantity.

as we expect stronger depolarization where the Faraday effects (rotation and dispersion) are larger. The depolarization is smallest (in absolute values) close to the minor axis growing in both directions along the major axis. Again, the area with low polarized signal (at about RA: $14^{\mathrm{h}} 53^{\mathrm{m}} 58^{\mathrm{s}}$; Dec: $+03^{\circ} 32^{\prime} 00^{\prime \prime}$ ) makes the depolarization very high there (low values), but - as in case of RM jumps - with large uncertainties.

As mentioned before, we inferred from the position of the dust lane and the X-ray distribution (Tüllmann et al. 2000, 2006) that the north-eastern side is the near side and the south-western is the far side of NGC 5775. Hence, we expect that the emission along the south-western side of the major axis is more affected by Faraday rotation and depolarization effects than the northeastern region along the major axis, which may explain the observed asymmetry in RM and depolarization perpendicular to the major axis.

\subsection{Magnetic field strength}

The magnetic field strength was calculated from the $4.86 \mathrm{GHz}$ data, assuming energy equipartition between cosmic-rays and magnetic fields (see Beck \& Krause 2005). We assumed a ratio of proton-to-electron energy density of 100. For a non-thermal spectral index in the extensions we assumed the value of $\alpha=$ 1.1 , which corresponds to the value in the spectral index map (Fig. 7) there, and a mean spectral index of $\alpha_{\mathrm{nt}}=0.92$ (as derived in Sect. 3.2) for the determination close to the disk plane. Assuming an axisymmetric magnetic field in the disk, close to the galactic disk we obtained values for the total and ordered magnetic field of $B_{\mathrm{t}}=11 \pm 3 \mu \mathrm{G}, B_{\text {reg }}=4 \pm 1 \mu \mathrm{G}$, respectively. In all four $X$-shaped extensions, we determined $B_{\mathrm{t}}=6 \pm 2 \mu \mathrm{G}$, $B_{\text {reg }}=4 \pm 1 \mu \mathrm{G}$. The total path length through the emitting 


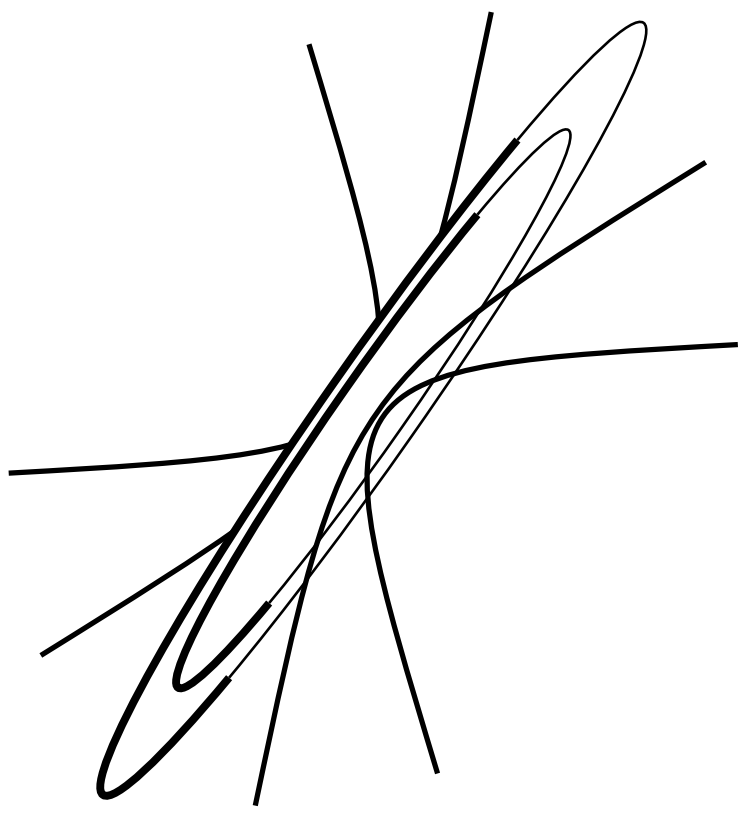

Fig. 12. Sketch of the large-scale magnetic field configuration in the disk and in the halo.

volume was adopted as $20 \mathrm{kpc}$ through the galactic midplane (see Fig. 6) and as $8 \mathrm{kpc}$ through the extensions. The magnetic field uncertainty takes into account uncertainties by a factor of two of the proton-to-electron ratio and the disk thickness.

With these values, the magnetic field strength in NGC 5775 is comparable to the field strength in NGC 253 (Heesen et al. 2009), which also hosts a nuclear starburst and strong star formation in the disk.

\subsection{Magnetic field structure}

The observed "apparent" magnetic vectors need to be corrected for Faraday rotation in order to derive the intrinsic magnetic field orientation. The Faraday rotation in NGC 5775 is $\leq \pm 30^{\circ}$ $\left(|\mathrm{RM}| \leq 140 \mathrm{rad} / \mathrm{m}^{2}\right)$ at $4.86 \mathrm{GHz}$. The corrected vectors are shown in Fig. 5. The orientations of the vectors is very similar to the observed $B$-vectors at $8.46 \mathrm{GHz}$ as the Faraday rotation is almost negligible $\left(\leq 10^{\circ}\right.$ for $\left.|\mathrm{RM}| \leq 140 \mathrm{rad} / \mathrm{m}^{2}\right)$ at this high frequency.

Close to the disk the magnetic orientation is plane-parallel. Magnetic vectors above $1 \mathrm{kpc}$ from the galactic disk, however, are dominated by vertical components forming an X-shaped structure (Fig. 5) as has been observed in several other edgeon galaxies (see e.g. Krause 2009). This pattern of the magnetic field vectors (presenting the direction of the magnetic field component projected on the sky-plane) together with the smooth and symmetric distribution of the RM distribution (Fig. 10) as a measure of the magnetic field strength along the line of sight shows that NGC 5775 hosts an ordered large-scale magnetic field configuration.

Figure 12 shows a sketch of the two predominant components of the large-scale magnetic field in NGC 5775, the planeparallel ASS disk field and the X-shaped halo field. The disk component seems to be highly asymmetric in its regularity. The maximum of polarized intensity is on the near side, shifted to the north-east and we see mostly emission from this field component. On the far side the magnetic field regularity is smaller and more of the synchrotron emission from the halo component can be seen. This situation is drawn in Fig. 12, where the line thickness illustrates the disk field regularity.

Such an asymmetry in magnetic field regularity is commonly seen in radio polarimetric observations of interacting galaxies (e.g. NGC 4254 Chyży et al. 2002). Indeed, NGC 5775 is known to interact with NGC 5774, a faint dwarf galaxy projected to the north-west of NGC 5775 (Irwin 1994; Gosh et al. 2009). There are two $\mathrm{H}_{\mathrm{I}}$ bridges with gas transfer from NGC 5774 to NGC 5775. Optical and radio emission was also detected along these bridges and Irwin 1994 suggested that this system may be in an early stage of a merger. This was supported by a recent analysis of X-ray data by Gosh et al. (2009) and may explain why the magnetic field regularity is still high along one side of NGC 5775.

\subsection{Dynamo action and galactic wind}

The optical spiral pattern in galaxies is usually trailing, and the magnetic field orientation is primarily parallel to the optical spiral arms as observed in all face-on spiral galaxies studied so far. If the direction of the disk rotation is known, one can use the RM-distribution to determine wether the magnetic field direction is inwards or outwards (Krause et al. 1989). The kinematics of NGC 5775 is well studied (e.g. Irwin 1994) with the northwestern side approaching and the south-eastern side receding. With the RM being predominantly negative on the approaching side we can conclude that the direction of the large-scale magnetic field in NGC 5775 is outwards. Together with NGC 4254 (Chyży 2008) and NGC 891 (Krause 2009) there are now three galaxies found with outwards directed ASS fields. The other four galaxies for which the direction of the ASS could be determined up to now (M 31, IC 342, NGC 253, and NGC 6949) were all inwards directed, which could not be explained up to now (Krause \& Beck 1998). The three counterexamples NGC 891, NGC 4254, and NGC 5775 balance the statistics as expected by theory which does not predict a preferred field direction.

A large-scale (unidirectional) magnetic field in a disk of the galaxy is generally thought to be dynamo-generated, e.g. by the mean field $\alpha \Omega$-dynamo. The magnetic field configuration that is most easily excited by this dynamo is the axisymmetric field (ASS) (e.g. Ruzmaikin et al. 1988). This field configuration consists of a strong unidirectional toroidal component with magnetic field lines running azimuthally within the disk and a weaker poloidal field with field lines looping perpendicularly to the azimuthal direction. The poloidal field can either be of quadrupole (separate loops above and below the plane-even-type) or dipolar (odd-type) symmetry (loops across the plane). In the even ASS-mode the magnetic field is symmetric with respect to the galactic mid-plane $(z=0)$ which is not the case for the odd mode (see Fig. 9 in Krause et al. 1989). Hence, in the even mode, the direction of the ASS disk field in the galactic disk is equal above and below the disk plane whereas in the odd mode it has opposite directions on both sides of the mid-plane.

For our case, the important consequence is the rotation measure signature for both modes, as seen in nearly edge-on disks. The even mode shows the antisymmetry with respect to the minor axis in the RM distribution and symmetry according to the major one. The odd mode shows antisymmetry with respect to both axes. As seen in Fig. 10 and as discussed in Sect. 4.4 the RM distribution has a clear asymmetry along the galactic midplane and no change of its sign across the plane. This and the fact that the field lines are oriented along the mid-plane agrees with an even axisymmetric disk field in the disk of NGC 5775 with 
magnetic field lines directed at the front side from south-east to north-west.

The accompanying quadrupolar poloidal field of this even ASS field configuration in the disk alone cannot explain the observed X-shaped field structure in NGC 5775, as it is - according to the mean-field dynamo theory - by a factor of about 10 weaker than the azimuthal disk field. However, model calculations of the mean-field $\alpha \Omega$-dynamo for a disk surrounded by a spherical halo including a galactic wind (Brandenburg et al. 1993; and Moss et al. 2010) simulated similar field configurations as the observed ones. Meanwhile, new MHD simulations of disk galaxies including a galactic wind are in progress and may explain the X-shaped field (Gressel et al. 2008; Hanasz et al. 2009a,b). The first global galactic-scale MHD simulations of a CR-driven dynamo give very promising results showing directly that magnetic flux is transported from the disk into the halo (Hanasz et al. 2009c).

Even for face-on galaxies with inclinations less than $60^{\circ}$, Braun et al. (2010) found evidence for a large-scale halo field from polarization asymmetries along the major axis which are consistent with a quadrupolar halo field and not with a dipolar halo field. As a quadrupolar halo field shows - at least in its inner part - a similar structure as an X-shaped magnetic field (which is different from a dipolar halo field) their results for face-on galaxies fit to our findings of X-shaped halo fields in edge-on galaxies.

The kinematics of ionized gas as well as the X-ray properties (Lehnert \& Heckman 1996; Tüllmann et al. 2006) suggest that NGC 5775 is even a "super-wind" galaxy. Such strong galactic winds from violent star formation areas should give rise to an almost spherical halo.

The large scale distribution of the various gas phases in the halo of NGC 5775 and its large scale magnetic field distribution is reminiscent of other galaxies with evidence for starburst driven winds such as NGC 253 (e.g. Heesen et al. 2009a,b) or NGC 4666 (e.g. Dahlem et al. 1997). The X-ray halo of NGC 5775 is well studied and its properties (Tüllmann et al. 2006; Li et al. 2008) corroborate the scenario for a "super-wind" from the inner disk (Strickland \& Stevens 2000) being present in this object. The examples of this class of objects mentioned above also have in common that the large scale magnetic field orientation in the halo has a characteristic X-shape. The distribution of the synchrotron radiation and its polarization characteristics result from the combination of the wind affecting the cosmic ray propagation and the magnetic field structure. The Xshaped structure of the wind results naturally from the centrifugal barrier of the effective galactic potential as has been shown by wind models for disk galaxies (e.g., Sushkov et al. 1998; Dalla Vecchia \& Schaye 2008). Unfortunately, all wind models so far neglect the influence of the magnetic field, which can only be included by full MHD simulations. A galactic wind may generally play an important role in the amplification of the largescale magnetic field by the mean-field dynamo, as it may solve the helicity problem of dynamo action (e.g. Sur et al. 2007).

\section{Summary and conclusions}

Deep polarized radio-continuum observations of the violently star forming galaxy $\mathrm{NGC} 5775$ at $8.46 \mathrm{GHz}$ together with archive data at lower frequencies allow us to reveal its global magnetic field configuration. We determined the total flux densities at all frequencies which fit well a single power-law with a spectral index of $\alpha=0.85 \pm 0.02$.
The rotation measure distribution varies smoothly on both sides along the major axis from positive values in the south-east to negative values in the north-west. The intrinsic magnetic field orientation along the galactic midplane is plane-parallel. From this we conclude that NGC 5775 has an even axisymmetric (even ASS) large-scale magnetic field configuration in the disk as generated by an $\alpha \Omega$-dynamo which is accompanied by a quadrupolar poloidal field. Taking into account informations about rotation of NGC 5775 we can conclude that the radial component of the plane-parallel magnetic field points outwards.

The intrinsic magnetic field pattern further away from the galactic plane forms an X-shaped structure as known from observations at lower frequency. Not only the magnetic field lines are X-shaped but also the distribution of the polarized intensity is $\mathrm{X}$-shaped. Four extensions are even indicated in the total power maps being accompanied with a small steepening of the spectral index.

The magnetic strengths were determined to be $B_{\mathrm{t}}=12 \pm$ $3 \mu \mathrm{G}$ for the total and $B_{\text {reg }}=4 \pm 1 \mu \mathrm{G}$ for the ordered field in the midplane (the ASS field). The field strength in the X-shaped structure reaches nearly the same values with $B_{\mathrm{t}}=9 \pm 2 \mu \mathrm{G}$ and $B_{\text {reg }}=4 \pm 1 \mu \mathrm{G}$. The field strength of the observed X-shaped halo magnetic field is by about one order of magnitude too high to be explained by the action of a mean-field dynamo alone. It may be due, however, to an interplay of the galactic wind in NGC 5775 together with dynamo action.

Acknowledgements. We thank the staff of the 100-m Effelsberg telescope for their assistance with the radio observations. This work benefited from the exchange program between the Ruhr-Universität Bochum (RUB) and the Jagiellonian University Kraków. Our thanks go to numerous colleagues at RUB and Astronomical Observatory of the Jagiellonian University for their valuable comments. We would like to thank the referee for his effort in improving the article. Support by grants from the Polish Government, grants no. PB3033/H03/2008/35 and 92/N-ASTROSIM/2008/0 as well as by Deutsche Forschungsgemeinschaft in the framework of SFB591 and FOR1048 at RUB is greatfully acknowledged.

\section{References}

Baars, J. W. M., Genzel, R., Pauliny-Toth, I. I. K., \& Witzel, A. 1997, A\&A, 61, 99

Beck, R., \& Krause, M. 2005, AN, 326, 414

Birk, G. T., Lesch, H., \& Neukirch, T. 1998, MNRAS, 296165

Brandenburg, A., Donner, K. J., Moss, D., et al. 1993, A\&A, 271, 36

Braun, R., Heald, G., \& Beck, R. 2010, A\&A, 514, A42

Chyży, K. T. 2008, A\&A, 482, 755

Chyży, K. T., Urbanik, M., Soida, M., \& Beck, R. 2002, Ap\&SS, 281, 409

Collins, J. A., \& Rand, R. J. 2001a, in Galaxy Disks and Disk Galaxies, ed. J. G.

Funes, \& E. M. Corsini (San Francisco), ASP Conf. Ser., 230, 307

Collins, J. A., \& Rand, R. J. 2001b, ApJ, 551, 57

Collins, J. A., Rand, R. J., Duric, N., \& Walterbos, R. A. M. 2000, ApJ, 536, 645

Dahlem, M., Lisenfeld, U., \& Golla, G. 1995, ApJ, 444, 119

Dahlem, M., Petr, M. G., Lehnert, M. D., et al. 1997, A\&A, 320, 731

Dahlem, M., Lisenfeld, U., \& Rossa, J. 2006, A\&A, 457, 121

Dalla Vecchia, C., \& Schaye, J. 2008, MNRAS, 387,1431

Dumke, M., \& Krause, M. 1998, in The Local Bubble and Beyond, ed. D. Breitschwerdt, M. Freyberg, \& J. Trümper, Proc. IAU Coll., 166, Lect. Notes Phys., 506 (Berlin: Springer-Verlag), 555

Dumke, M., Krause, M., Wielebinski, R., \& Klein, U. 1995, A\&A, 302, 691

Duric, N., Irwin, J., \& Bloemen, H. 1998, A\&A, 331, 428

Emerson, D. T., \& Gräve, R. 1988, A\&A, 190, 353

Elstner, D., Meinel, R., \& Beck, R. 1992, A\&AS, 94, 587

Golla, G., \& Hummel, E. 1994, A\&A, 284, 777

Gosh, K. K., Saripalli, L., Gandhi, P., et al. 2009, ApJ, 137, 3263

Gressel, O., Ziegler, U., \& Rüdiger, G. 2008, A\&A, 486, L35

Hanasz, M., \& Lesch, H. 2003, A\&A, 412, 331

Hanasz, M., Otmianowska-Mazur, K., Kowal, H., \& Lesch, H. 2009a, A\&A, 498, 335 
Hanasz, M., Otmianowska-Mazur, K., Lesch, H., et al. 2009b, in IAU Symp., 259,479

Hanasz, M., Wóltański, D., \& Kowalik, K. 2009c, ApJ, 706, L155

Heesen, V., Beck, R., Krause, M., \& Dettmar, R.-J. 2009a, A\&A, 494, 563

Heesen, V., Krause, M., Beck, R., \& Dettmar, R.-J. 2009b, A\&A, 506, 1123

Irwin, J. A. 1994, ApJ, 429, 618

Krause, M. 2009, in Magnetic Fields in the Universe II, ed. A. Esquirel, Rev. Mex. Astron. Astrofis., 36, 25

Krause, F., \& Beck, R. 1998, A\&A, 335, 786

Krause, M., Hummel, E., \& Beck, R. 1989, A\&A, 217, 4

Lee, S.-W., Irwin, J. A., Dettmar, R.-J., et al. 2001, A\&A, 377, 759

Lee, S.-W., Seaquist, E. R., Leon, S., García-Burillo, S., \& Irwin, J. A. 2002, ApJ, 573, L107

Lehnert, M. D., \& Heckman, T. M. 1996, ApJ, 472, 546

Li, J. T., Li, T., Wang, D., et al. 2008, MNRAS, 390, 59
Moss, D., Sokoloff, D., Beck, R., \& Krause, M. 2010, A\&A, 512, A61

Rand, R. J. 2000, ApJ, 537, L13

Rossa, J., \& Dettmar, R.-J. 2000, A\&A, 359, 433

Ruzmaikin, A. A., Shukurov, A. M., \& Sokoloff, D. D. 1988, Magnetic Fields of Galaxies (Dordrecht: Kluwer)

Shukurov, A., Sokoloff, D., Subramanian, K., et al. 2006, A\&A, 448, L33

Strickland, D. K., \& Stevens, I. R. 2000, MNRAS, 314, 511

Suchkov, A. A., Berman, V. G., Heckman, T. M., et al. 1998, ApJ, 463, 528

Sur, S., Shukurov, A., \& Subramanian, K. 2007, MNRAS, 377, 874

Tabatabaei, F. S., Beck, R., Krügel, E., et al. 2007, A\&A, 475, 133

Taylor, A. R., Stil, J. M., \& Sunstrum, C. 2009, ApJ, 702, 1230

Tüllmann, R., Dettmar, R.-J., Soida, M., Urbanik, M., \& Rossa, J. 2000, A\&A, 364, L36

Tüllmann, R., Pietsch, W., Rossa, J., Breitschwerdt, D., \& Dettmar R.-J. 2006, A\&A, 448, 43 